\title{
The Status of the Acupuncture Mechanism Study Based on PET/ PET-CT Technique: Design and Quality Control
}

\author{
Zhaoxuan He $\mathbb{D}^{1,2}$ Likai Hou, ${ }^{1,2}$ Ruirui Sun, ${ }^{1,2}$ Tao Yin $\mathbb{D}^{1,2}$ Peihong Ma, ${ }^{1,2}$ Li Chen $\mathbb{D}^{1,2}$ \\ Shirui Cheng $\mathbb{D},{ }^{1,2}$ Xiaoyan Liu $\mathbb{D}$, , ${ }^{1,2}$ Xiaojuan Hong $\mathbb{D}{ }^{1,2}$ Jian Hou $\mathbb{D},,^{2,3}$ and Fang Zeng $\mathbb{D}$ 1,2 \\ ${ }^{1}$ Acupuncture and Tuina School/The 3rd Teaching Hospital, Chengdu University of Traditional Chinese Medicine, Chengdu, \\ Sichuan, China \\ ${ }^{2}$ Acupuncture \& Brain Science Research Center, Chengdu University of Traditional Chinese Medicine, Chengdu, \\ Sichuan, China \\ ${ }^{3}$ Clinical Medicine College/Teaching Hospital, Chengdu University of Traditional Chinese Medicine, Chengdu, Sichuan, China \\ Correspondence should be addressed to Jian Hou; hoj2000@126.com and Fang Zeng; zeng_fang@126.com
}

Received 27 June 2019; Accepted 31 October 2019; Published 29 November 2019

Academic Editor: Luciana Dini

Copyright ( 2019 Zhaoxuan He et al. This is an open access article distributed under the Creative Commons Attribution License, which permits unrestricted use, distribution, and reproduction in any medium, provided the original work is properly cited.

\begin{abstract}
$\mathrm{PET} / \mathrm{PET}-\mathrm{CT}$ is an important technique to investigate the central mechanism of acupuncture in vivo. This article collected original research papers with keywords of "Acupuncture," "PET," "PET/CT," and "Positron emission tomography" in PubMed and CNKI databases from January 2003 to December 2018. As a result, a total of 43 articles were included. Based on the literature analyses, we found that (1) reasonable arrangement of the operation process and the choice of appropriate acupuncture intervention time is conducive to a better interpretation of acupuncture-PET/PET-CT mechanism and (2) the selection of participants, sample size, acupuncture intervention, and experimental conditions would affect study results. Therefore, effective quality control is an important way to ensure the repeatability of research results.
\end{abstract}

\section{Introduction}

Acupuncture, as an essential component of traditional Chinese medicine (TCM), is increasingly accepted as an alternative and complementary therapy by western countries for its reliable effect of relieving pain and regulating visceral functions [1-3]. Since the 1970s, a number of studies have confirmed that the central nervous system (CNS) plays an important role in acupuncture effect $[4,5]$. With the development of neuroimaging techniques, using functional Magnetic Resonance Imaging (fMRI) and Positron Emission Tomography (PET) to investigate the cerebral responses to acupuncture stimulation in vivo has gradually become a spotlight in acupuncture mechanism research. After analyzing the acupuncture-neuroimaging articles published from 1995 to 2014, we found that MRI and PET/PET-CT are the two imaging technologies most commonly used [6].

PET, as the representative of biochemical imaging, becomes an important technique to explore the acupuncture mechanism for its higher functional resolution and significant integrity $[7,8]$. Combining the functional metabolic imaging and anatomical imaging, positron emission tomographycomputed tomography (PET-CT) stands for the highest level of current nuclear medical imaging techniques. It can clearly reflect the change of corresponding functional areas of the brain after stimulus, so PET/PET-CT has been widely used in brain research studies [9]. In the past two decades, many investigators had mapped the central activity changes elicited by acupuncture stimulation using PET/PET-CT which provided visualized and reliable interpretation for acupuncture treating stroke, depression, and other diseases [10-12].

However, the study designs, especially the scanning procedures of these acupuncture-PET/PET-CT studies, have significant differences. To our knowledge, there are at least 6 kinds of scanning procedures used in the acupuncture study. The methodological differences limit the further application of PET/PET-CT in acupuncture studies and affect the repeatability and reliability of the results of these studies. 
So, this study aims to analyze the status of the acupuncture-PET/PET-CT studies from the study design and the quality control by comparing the original articles from 2003 to 2018, so as to provide references for future studies.

\section{Methods}

2.1. Searching Strategy. We searched China National Knowledge Infrastructure (CNKI, 1979-2018) for articles from January 1, 2003, to December 31, 2018, in Chinese language by the title/abstract search. The detailed search terms and search strategies are as follows: ("Acupuncture" (Zhenjiu) OR "Needling" (Zhenci) OR "Manual acupuncture" (Shouzhen) OR "Electroacupuncture" (Dianzhen) OR "Scalp acupuncture" (Touzhen)) AND ("PET" OR "PET/CT" OR "Positron emission tomography" OR "Positron emission tomography with computed tomography"). Also, we searched the original articles published from January 1, 2003, to December 31, 2018, in PubMed (1959-2018) using the following Mesh terms and search strategies:("Acupuncture"[Mesh terms] OR "Acupuncture Therapy"[Mesh terms] OR “Acupuncture, Ear" OR "Acupuncture Points"[Mesh terms] OR “Acupuncture Analgesia”[Mesh terms]) AND ("Positron emission tomography"[Mesh terms]).

We screened the bibliographies of identified trials and reviewed articles for further potentially relevant publication. Subsequently, we screened the full texts and assessed whether these articles met the inclusion criteria.

2.2. Inclusion and Exclusion Criteria. The articles would be included, if they were: (1) original articles; (2) acupuncturePET/PET-CT study on human beings; (3) published in English or Chinese; and (4) published from January 1, 2003, to December 31, 2018.

The articles would be excluded, if they were: (1) reviews, case reports, editorials, trial protocols, or letters; (2) acupuncture-PET/PET-CT study on animals; or (3) duplicate articles. (Figure 1 shows the flowchart of the literature selection).

2.3. Data Extraction and Analysis. We extracted the data including characteristics of the participants (patients or the health, accompanying symptoms), sample size, acupuncture intervention (method of intervention, manipulation procedure, Deqi/needle sensation, and acupuncturist), PET/ PET-CT scanning process, and imaging agent types. Data analysis was conducted after data extraction.

\section{Results}

Forty-three original articles were included.

3.1. Annual Distribution of the Studies. The annual distribution of the studies was shown in Figure 2. In 2012, the acupuncture studies using PET/PET-CT reached the peak.
3.2. Classification of Participants. Fourteen studies were performed on healthy subjects [13-26]. Twenty-three studies were performed on patients, involving 10 diseases in 4 systems [10-12, 27-46]. Six studies recruited both healthy subjects and patients [47-52]. (Figure 3).

3.3. Sample Size. The average sample size of these studies was 8 participants per group. There is no difference in sample size between the studies performed on healthy subjects and patients.

\subsection{Acupuncture Intervention}

3.4.1. Acupuncture Modalities. Nineteen studies chose manual acupuncture as the intervention method $[10,13$, 20-25, 28, 29, 32, 33, 35-37, 39, 43, 46, 47]. Twenty-four studies chose electroacupuncture as the intervention method $[11,12,14-19,26,27,30,31,34,38,40-42,44,45,48-52]$.

3.4.2. Manipulation Procedure. Except 1 paper which did not describe the acupuncture manipulation [32], the other 42 articles described the manipulation procedure of acupuncture.

3.4.3. Deqi (Needle Sensation). Thirty-three studies emphasized the needle sensation (Deqi) during acupuncture stimulation [11, 13-20, 22, 23, 26-31, 34, 36-42, 44, 46-52].

Two studies evaluated the needle sensation with the 10point Visual Analogue Scale (VAS) and the Needle Sensation Questionnaire (NSQ) [22, 46].

3.4.4. Qualification of Acupuncturists. Fourteen articles mentioned the qualification of acupuncturists $[13,15,16,20,21,23,24,27,30,33,39,41,43$, 47].

3.5. PET/PET-CT Scanning Process. The PET/PET-CT scanning processes can be divided into two categories: (1) acupuncture and PET/PET-CT scan are not on the same day (Figure 4, Model (1) $[11$, 12, 28, 29, 32, 34, 35, 37, 39, 42, 44-46, 48, 51] And (2) acupuncture and PET/ PET-CT scan are on the same day (Figure 4, Model (2) $[10,13-27,30,31,33,36,38,40,41,43,47,49,50,52]$.

According to the timing of acupuncture stimulation in the scanning process, the Model 2 can be divided into two subtypes: scanning with acupuncture stimulation (Figure 4, Model 2A) [15-17, 25, 26, 43, 49, 50, 52] and scanning after acupuncture stimulation (Figure 4, Model 2B) $[10,13,14,18-24,27,30,31,33,36,38,40,41,47]$. According to the sequence of acupuncture stimulation and tracer injection, the Model 2A can be divided into two subtypes: injecting after inserting needle (Figure 4, Model 2A-1) $[25,43,49,50,52]$ and injecting before inserting needle (Figure 4, Model 2A-2) [15-17, 26]. The Model 2B can be divided into three subtypes: injecting during inserting needle (Figure 4, Model 2B-1) [14, 18, 30, 38], injecting after inserting needle (Figure 4, Model 2B-2) [10, 13, 19, 20, 


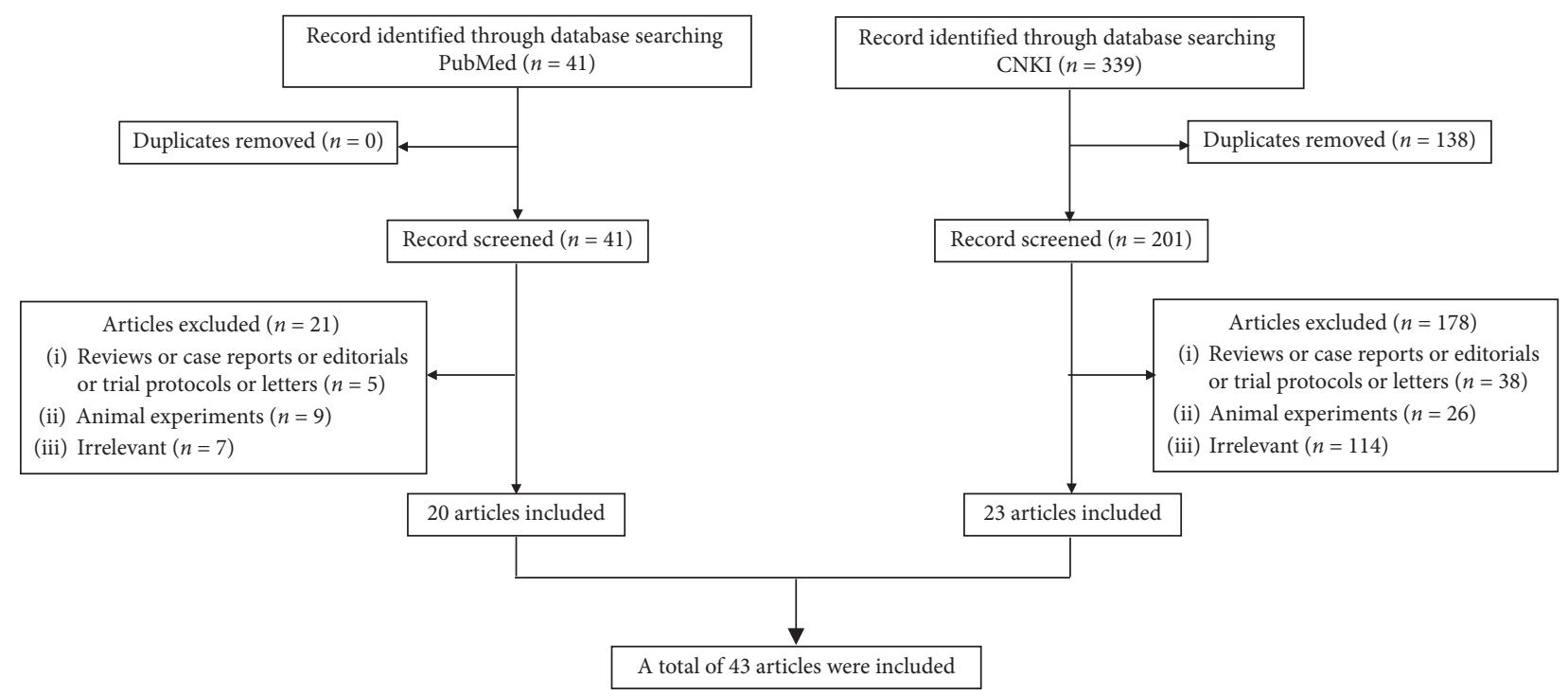

FIgURE 1: The flowchart of literature search and screening process.

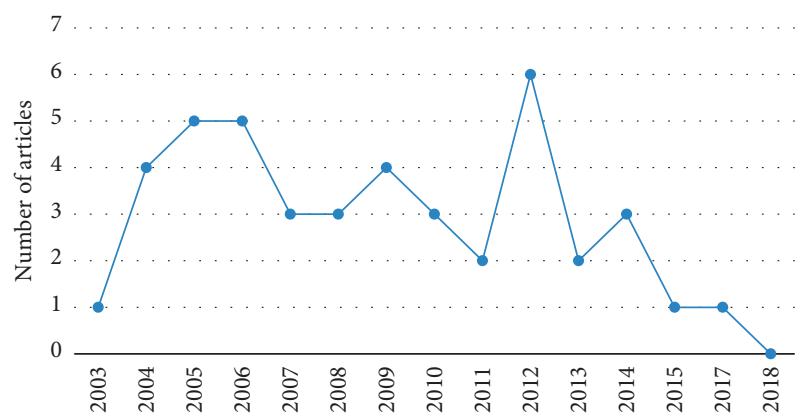

Figure 2: Annual distribution of the acupuncture-PET/PET-CT studies.

$22-24,27,31,33,36,40]$, and injecting before inserting needle (Figure 4, Model 2B-3) [21, 41, 47].

Studies of Model 2 were the largest proportion (65\%), and studies of Model 1 accounted for about 35\% (Figure 5).

3.6. Imaging Agent Types. Among the imaging agents, fluorine-18 fluorodeoxyglucose(18F-FDG) (95\%) was most commonly used in the PET/PET-CT scanning, and only two papers used labeled water $(5 \%)[25,46]$.

\section{Discussion}

As a main neuroimaging technique, PET/PET-CT shows an irreplaceable advantage in biochemical imaging and becomes an important method to explore the central mechanism of acupuncture. Using PET/PET-CT, investigators had mapped the central responses to acupuncture treatment not only on healthy subjects but also on patients with neuropathy, psychological disorders, gastrointestinal disorders, and pain (Figure 3).

In this study, we found that, from 2003 to 2012, the number of acupuncture-PET/PET-CT studies increased and peaked in 2012, but decreased significantly thereafter. The reasons for this decline might be due to its expensive cost, non-negligible radiation risk, and unregular experimental design.

With the advent of the hybrid PET/MR and its gradual application in clinic and research, people have begun to try to apply it in acupuncture research. So, it is necessary to review the methodological issues in the acupuncture-PET studies, so as to provide reference for optimizing the future study. Among these methodological issues involved in the study design, there are two main aspects in the disagreement in these studies. One is the process of PET/PET-CT scan, and the other is the quality control-related information.

4.1. The Process of PET/PET-CT Scan. Owing to different experimental condition and purpose, the PET/PET-CT scanning process was quite different. There are at least six kinds of scan processes recorded in these papers. This difference is heightened in the timing of acupuncture stimulation.

4.1.1. The Types of PET/PET-CT Scanning Process. In the study, the PET/PET-CT scanning processes were divided according to three aspects: (1) whether the acupuncture stimulation was performed on the same day as the PET/PETCT scan. According to this, the scanning processes were divided into two models: Model 1, acupuncture and PET/ PET-CT scan were not performed on the same day and Model 2, acupuncture and PET/PET-CT scan were performed on the same day. (2) Whether the scanning is performed simultaneously with acupuncture stimulation. According to this, the Model 2 was divided into two types: Model 2A is scanning with acupuncture stimulation, and Model $2 \mathrm{~B}$ is scanning after acupuncture stimulation. (3) What is the timing of acupuncture stimulation and tracer injection? According to this, the Model 2A was divided into two subtypes (Model 2A-1 and Model 2A-2), and the Model 


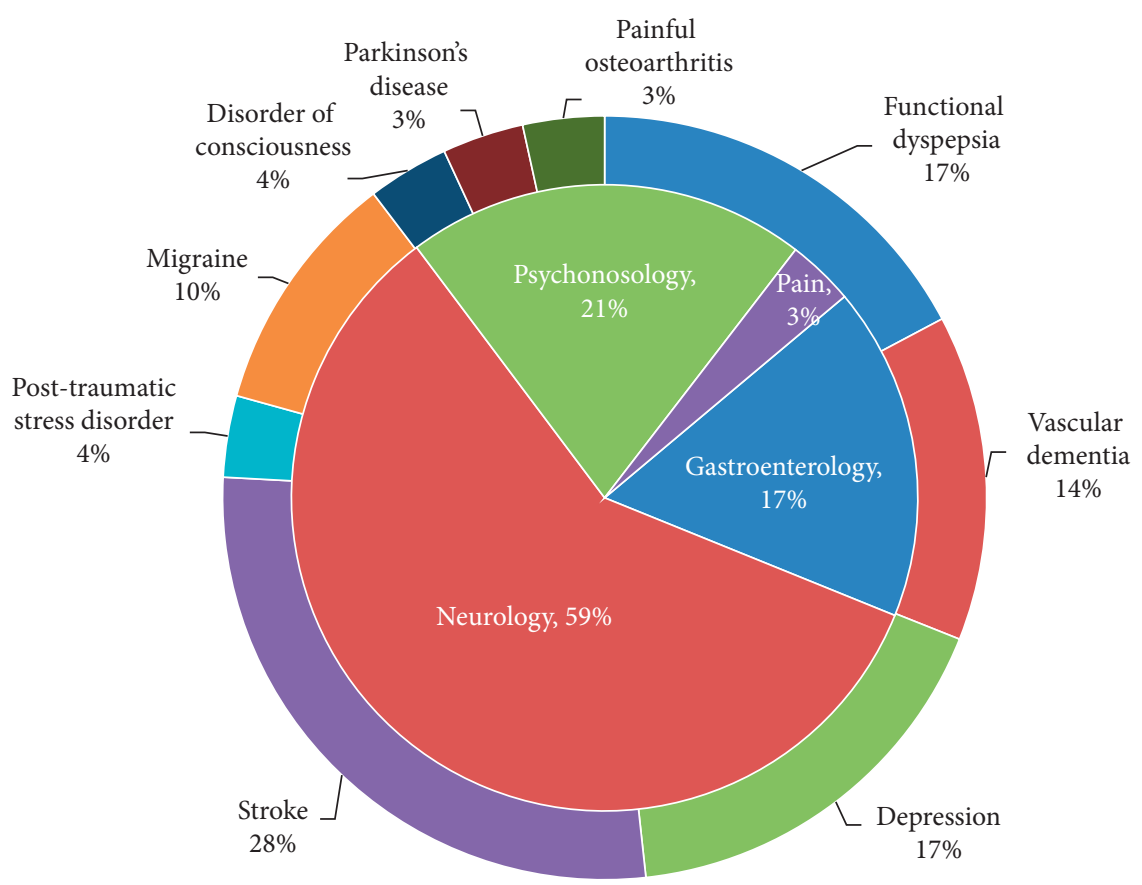

FIgURE 3: The distribution of disease system and disease category involved in acupuncture-PET/PET-CT studies.

Model 1 Acupuncture and PET/PET-CT scan are not on the same day

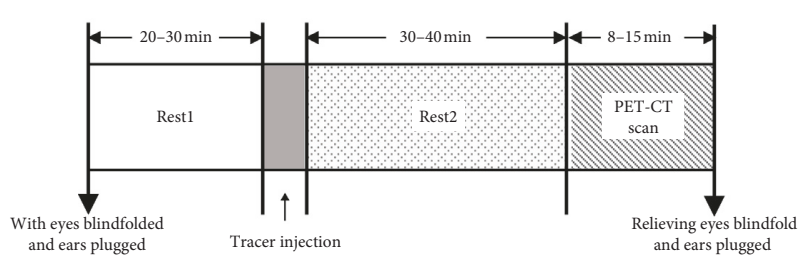

Model 2 Acupuncture and PET/PET-CT scan are on the same day Model 2A Scanning with acupuncture stimulation

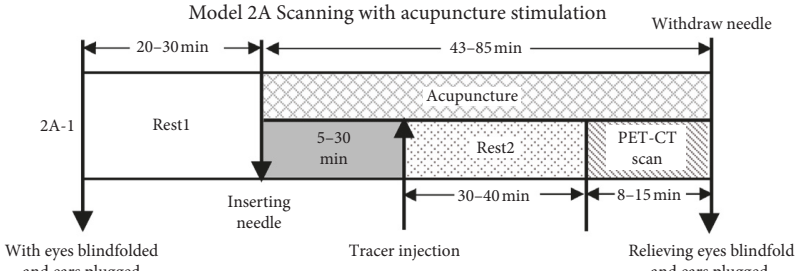
and ears plugged

Model 2A-1 Needle in-injection-scanning-needle out

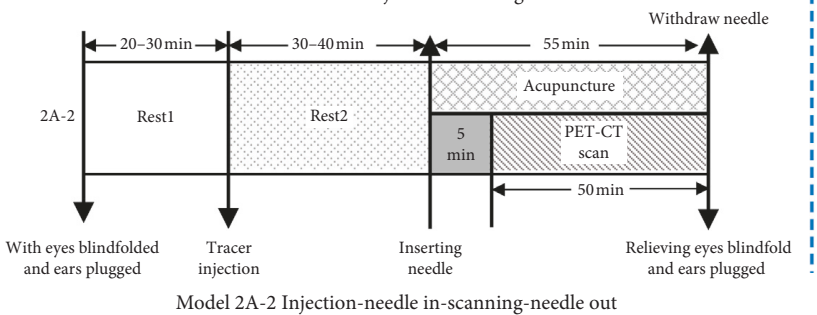

Model 2B Scanning after acupuncture stimulation

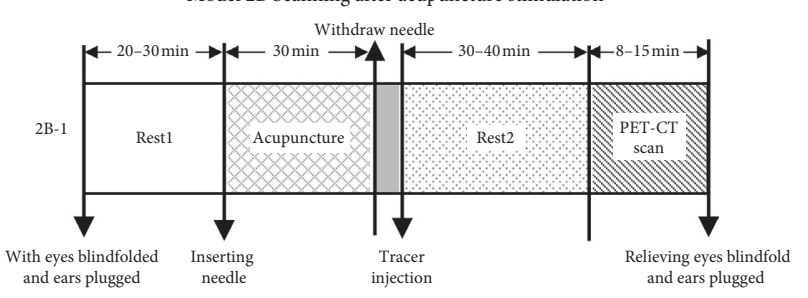

Model 2B-1 Needle in-needle out-injection-scanning

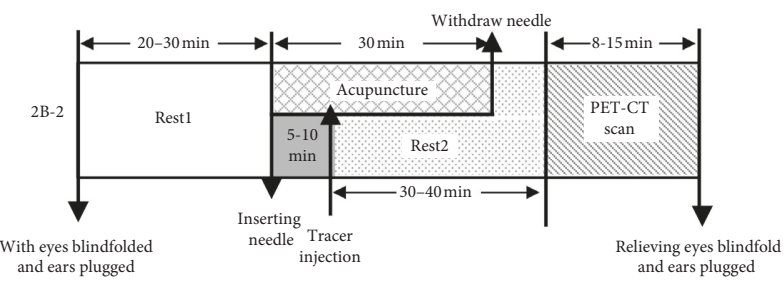

Model 2B-2 Needle in-injection-needle out-scanning Tracer injection

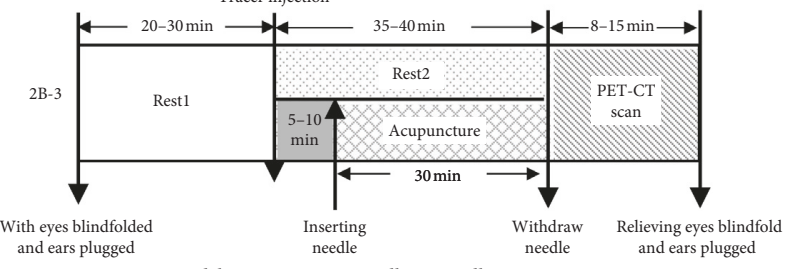

Model 2B-3 Injection-needle in-needle out-scanning

FIgure 4: The models of PET/PET-CT scan.

2B was divided into three subtypes (Model 2B-1, Model 2B2, and Model 2B-3). (Figures 4 and 5).

4.1.2. The Characteristics of Each Type. The main feature of Model 1 is to scan completely in a resting state. It follows the routine scan process including resting, tracer injection, resting, and then scanning (Figure 4). The advantage of this model is that there is less interference in the scanning process.

The main characteristic of Model 2 is that the scanning and acupuncture stimulations are performed on the same day. 


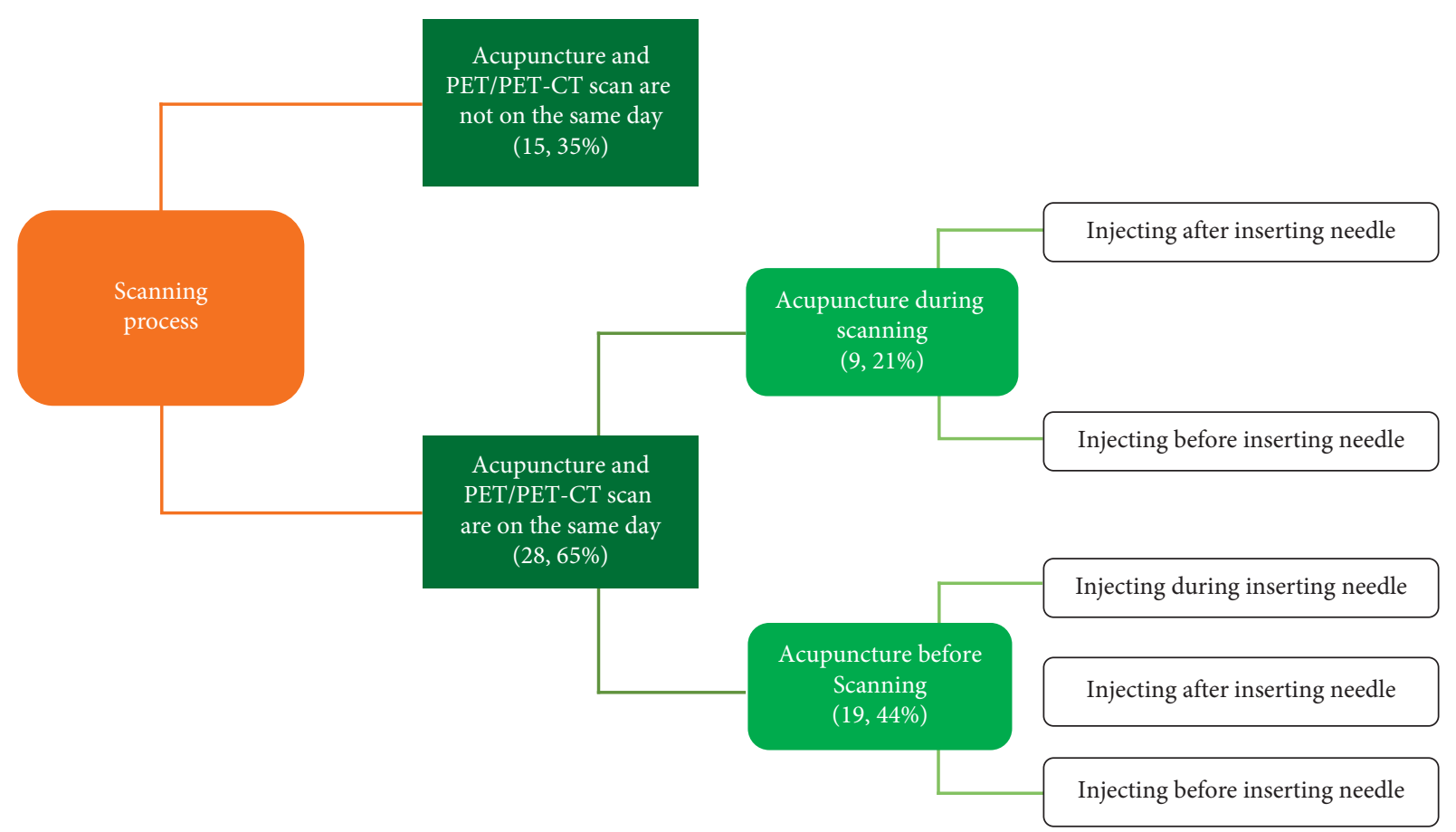

FIgURe 5: PET/PET-CT scanning process classification.

For Model 2A, it is marked by scanning and acupuncture stimulation at the same time. Its advantage is that it can achieve the most significant cerebral responses to acupuncture stimulation. However, scanning with acupuncture needles retained in the body might bring some disturbing factors and risk factors. The Model 2A is mainly applied to investigate the central mechanism of the instant effect of acupuncture stimulation on the patients with paroxysmal diseases or healthy subjects.

The main differences between Model 2A-1 and Model 2A2 are the timing of acupuncture needle insertion and tracer injection and the retaining time of the needles. Compared with Model 2A-2, Model 2A-1 shows longer retaining time of the needles. In some study, the retaining time is even more than 1 hour. It is not very consistent with clinical practice.

For Model 2B, it is characterized by scanning after acupuncture stimulation. Its advantage lies in not only gaining the obvious cerebral responses elicited by acupuncture but also avoiding the interference of acupuncture manipulation on scanning.

The main difference among these three subtypes of model $2 \mathrm{~B}$ is also the time of acupuncture stimulation and tracer injection. In details, in Model 2B-1, the tracer injection is performed just after withdrawing the acupuncture needle (acupuncture stimulation is over). The order of acupuncture and tracer injection is needle in-needle out-tracer injection. In Model 2B-2, the tracer injection is performed during the period of acupuncture stimulation. The order of acupuncture and tracer injection is needle in-tracer injection-needle out. In Model 2B-3, the tracer injection is performed before inserting the acupuncture needle (acupuncture stimulation is begun). The order of acupuncture and tracer injection is tracer injection-needle in-needle out.
4.1.3. The Reasons of These Different Designs. The reasons of these different designs can be analyzed from 3 aspects.

Firstly, whether the acupuncture stimulation and PET/ PET-CT scan are performed on the same day is decided by the purpose of study. The Model 1 is applied to explore the mechanism of the long-term effect of acupuncture treatment, while the Model 2 is more suitable for investigating the instant effect of acupuncture.

Secondly, whether the scanning and acupuncture stimulation are performed at the same time depends on a comprehensive assessment of the participants' physical conditions, potential risk, and interference factor.

Thirdly, the issue of who is the first to inject the tracer and to insert needles reflects two considerations of investigators. One is the half-life of the tracer. For example, the half-life of $18 \mathrm{~F}-\mathrm{FDG}$ is 109.8 minutes. It is reported that after entering the body for 40 minutes, its metabolism tends to stabilize [53]. So, the PET/PET-CT scan should be performed after injection of 18F-FDG at 35-40 minutes. For the same reason, it is better to perform acupuncture stimulation after 35-40 minutes by injecting 18F-FDG. In this way, it can make the responsive process of acupuncture signal in the brain basically coincide with the process of $18 F-F D G$ metabolism stabilizing in the brain. The other is whether the sharp pain induced by the tracer injection significantly influences cerebral activity. Some investigators hold that the sharp pain induced by the tracer injection is stronger than the needle sensation, and it can elicit significant cerebral activity changes and result in false positive results, so they selected inserting needles after tracer injection [54]. Compared to Model 2B-1 and Model 2B-2, Model 2B-3 takes these two aspects mentioned above into consideration. 
4.2. The Quality Control. Due to the complexity of the brain function and the variety of acupuncture manipulations, the selection of participants, sample size, acupuncture intervention, and experimental condition would affect the results of the study. Therefore, seeking a more reasonable and practical method to improve the reproducibility and reliability of results seems to be important to acupunctureneuroimaging study.

4.2.1. The Selection of Participants. Based on the literature analyses, we found that the majority of PET/PET-CT studies (55\%) were performed on patients. According to the theory of traditional Chinese acupuncture, acupuncture treatment acts on strengthening the body resistance to removing pathogenic factors, and the efficacy of acupuncture treatment is specific to the pathological state, not the physiological conditions [55]. The function of acupoint is body condition dependent, and the acupoint is more active during the pathological conditions than in a healthy state $[56,57]$. So, patients should be a better choice for acupunctureneuroimaging studies [6]. In this study, we also found that the diseases of the nervous system were the most common choice in the acupuncture-PET/PET-CT study. The result indicated that acupuncture has a good effect for diseases of the nervous system, such as stroke and depression [10-12]. However, acupuncture does better in regulating functional disorders rather than curing organic diseases [58]. More attention could be paid on the mechanism of acupuncture regulating functional disorders.

In addition, the subtypes of disease should be taken into consideration when you choose patients as the participants in the neuroimaging study. Patients with different subtypes might have cerebral functional or/and structural differences. In previous studies, we compared with epigastric pain syndrome (EPS), and acupuncture has a more significant effect in the treatment of FD in patients with postprandial distress syndrome (PDS) [59]. So, in acupuncture-neuroimaging study, the same subtype of a disease can be chosen to ensure the homogeneity of participants and reduce the baseline state of brain function of subjects.

4.2.2. Sample Size. Due to the potential radioactivity and the costs of imaging, the sample size in most of PET/PET-CT studies was small. Most studies controlled sample size between 6 and 15 cases. In this study, we found that the average sample size was 8 participants per group, no matter the healthy subjects and patients. Some researchers hold that the activated brain regions are related to the sample size [60]. Nowadays, most investigators agreed that a bigger sample size brought the achievement of stable statistical power [61].

4.2.3. Acupuncture Intervention. As we know, the defined influence of the qualification of the acupuncturist and manipulation procedure has an effect on clinical efficacy. In clinical studies, we should formulate the standard process of the acupuncture operation that included numbers of needle, depth of insertion, elicited response, and needle retention time. Practitioners should choose a qualified professional acupuncturist and the same acupuncturist in one research as far as possible.

Deqi (needle sensation) plays an important role in acupuncture efficacy. A majority of clinical trials have demonstrated that the curative effect with needle sensation was superior to those without needle sensation [62]. A neuroimaging study also showed a significant difference in cerebral responses under the Deqi and non-Deqi condition [63]. So, it is important to require Deqi in acupunctureneuroimaging studies.

However, we found that the questionnaire-based forms were less used to assess the needle sensation in acupuncturePET-CT studies. There were only two kinds of forms such as 10-point Visual Analogue Scale (VAS) and Needle Sensation Questionnaire (NSQ) applied to these researches.

4.2.4. Experimental Condition. In brain imaging studies, the individual's psychological activity, the voice, and light of the external environment would lead to the change of brain activity, so we should strictly control each step of the experimental preparation, such as audio-visual closed, rest before the test, and acupuncture preparation. As far as possible, we ought to unify the time of acupuncture therapy and PET/PET-CT scans.

\section{Conclusion}

This paper focuses on the scanning process and experimental quality control to analyze the methodological issues involved in the acupuncture-PET/PET-CT studies in detail. The results provide useful references for future PET/PET-CT study and even PET-MR study.

\section{Disclosure}

Zhaoxuan He, Likai Hou, and Ruirui Sun are co-first authors and contributed equally to this paper.

\section{Conflicts of Interest}

The authors declare that they have no conflicts of interest.

\section{Authors' Contributions}

Zhaoxuan He, Likai Hou, and Ruirui Sun designed the study and drafted the manuscript. Fang Zeng and Jian Hou revised the study design and the manuscript. Zhaoxuan He, Tao Yin, Peihong Ma, and Xiaoyan Liu participated in the design of the search strategy and data extraction. Likai Hou, Li Chen, Shirui Cheng, and Xiaojuan Hong formed the data synthesis and analysis plan. All authors have read and approved the publication of the final manuscript.

\section{Acknowledgments}

This work was supported by the National Natural Science Foundation of Outstanding Youth Fund (grant no. 
81622052) and Ten Thousand Talent Program (grant no. W02020595).

\section{References}

[1] T. Takahashi, "Mechanism of acupuncture on neuromodulation in the gut-a review," Neuromodulation: Journal of the International Neuromodulation Society, vol. 14, no. 1, pp. 8-12, 2011.

[2] Q.-L. Yuan, P. Wang, L. Liu et al., "Acupuncture for musculoskeletal pain: a meta-analysis and meta-regression of sham-controlled randomized clinical trials," Scientific Reports, vol. 6, no. 1, Article ID 30675, 2016.

[3] A. J. Vickers, E. A. Vertosick, G. Lewith et al., "Acupuncture for chronic pain: update of an individual patient data metaanalysis," The Journal of Pain, vol. 19, no. 5, pp. 455-474, 2018.

[4] L. Bai, W. Qin, J. Tian et al., "Acupuncture modulates spontaneous activities in the anticorrelated resting brain networks," Brain Research, vol. 1279, pp. 37-49, 2009.

[5] K. K. S. Hui, J. Liu, N. Makris et al., "Acupuncture modulates the limbic system and subcortical gray structures of the human brain: evidence from fMRI studies in normal subjects," Human Brain Mapping, vol. 9, no. 1, pp. 13-25, 2000.

[6] K. Qiu, M. Jing, R. Sun et al., "The status of the quality control in acupuncture-neuroimaging studies," Evidence-Based Complementary and Alternative Medicine: eCAM, vol. 2016, Article ID 3685785, 14 pages, 2016.

[7] D. W. Townsend, J. P. J. Carney, J. T. Yap et al., "PET/CT today and tomorrow," Journal of Nuclear Medicine: Official Publication, Society of Nuclear Medicine, vol. 45, no. 1, pp. 4S-14S, 2004.

[8] F. Zeng, X.-G. Liu, Y. Tang et al., "Application of PET-CT technique to the research on central mechanism of acupuncture effects," Acupuncture Research, vol. 33, no. 4, pp. 284-286, 2008.

[9] S. Chinchure, C. Kesavadas, and B. Thomas, "Structural and functional neuroimaging in intractable epilepsy," Neurology India, vol. 58, no. 3, pp. 361-370, 2010.

[10] Y. Huang, C. Tang, S. Wang et al., "Acupuncture regulates the glucose metabolism in cerebral functional regions in chronic stage ischemic stroke patients---a PET-CT cerebral functional imaging study," BMC Neuroscience, vol. 13, no. 1, p. 75, 2012.

[11] Y. Huang, D.-J. Li, A.-W. Tang et al., "Effect of scalp acupuncture on glucose metabolism in brain of patients with depression," Zhongguo Zhong Xi Yi Jie He Za Zhi, vol. 25, no. 2, pp. 119-122, 2005.

[12] Z. Fang, J. Ning, C. Xiong et al., "Effects of electroacupuncture at head points on the function of cerebral motor areas in stroke patients: a PET study," Evidence-Based Complementary and Alternative Medicine: eCAM, vol. 2012, Article ID 902413, 2012.

[13] X.-S. Lai, Y. Huang, J.-Q. Chen et al., "Using PET brain function imaging technique to study the activation effect of acupuncture at Waiguan on brain functional area," Journal of Sichuan of Traditional Chinese Medicine, vol. 27, no. 3, pp. 104-106, 2009.

[14] Z.-Z. Jin, "Analysis of the effects of Mongolian medicine acupuncture on the changes of brain metabolism based on PET-CT brain function imaging," Chinese Journal of Medicine, vol. 20, no. 3, pp. 18-20, 2014.

[15] G.-r. Shapo, C. Liu, G.-B. Wang et al., "Localizing the specific functional areas within the human brain of acupuncture the acupoint BL40 (Weizhong) with functional PET-CT imaging," in Proceedings of the 2nd Medical Imaging Shandong Forum Proceedings, Shandong, China, 2005.

[16] G.-r. Shapo, L. Ma, C. Liu et al., "Effects of acupuncture at Weizhong (BL40) on brain function with PET/CT," Zhongguo Zhong Xi Yi Jie He Za Zhi, vol. 26, no. 11, pp. 969-972, 2006.

[17] G.-r. Shapo, B. Yan, C. Liu et al., "Acupuncture of Weizhong (BL40) and Zusanli (ST36) on the study of brain function by PET/CT," Chinese Journal of Nuclear Medicine, vol. 26, no. 1, pp. 54-56, 2006.

[18] X.-Z. Meng, J. Zhang, J.-W. Zhang et al., "Effects of electroacupuncture at acuponit quchi on cerebral functional imaging by PET-CT," Shanghai Journal of Traditional Chinese Medicine, vol. 42, no. 12, pp. 42-44, 2008.

[19] P. Gong, M.-M. Zhang, L.-M. Jiang et al., "Research on effect of acupuncture at Sanyinjiao on brain function by means of positron emission tomographic imaging," Zhongguo Zhong Xi Yi Jie He Za Zhi, vol. 26, no. 2, pp. 119-122, 2006.

[20] G.-F. Zhang, Y. Huang, C.-Z. Tang et al., "Effect of psychological comfort on acupuncture analyzing by PET-CT functional brain imaging," Liaoning Journal of Traditional Chinese Medicine, vol. 44, no. 1, pp. 128-223, 2017.

[21] M.-S. Park, Y.-Y. Sunwoo, K.-S. Jang et al., "Changes in brain FDG metabolism induced by acupuncture in healthy volunteers," Acta Radiologica, vol. 51, no. 8, pp. 947-952, 2010.

[22] G.-F. Zhang, Y. Huang, C.-Z. Tang et al., "Characteristics of PET cerebral functional imaging during "Deqi" of acupuncture in healthy volunteers," Acupuncture Research, vol. 36, no. 1, pp. 46-51, 2011.

[23] X. Lai, G. Zhang, Y. Huang et al., "A cerebral functional imaging study by positron emission tomography in healthy volunteers receiving true or sham acupuncture needling," Neuroscience Letters, vol. 452, no. 2, pp. 194-199, 2009.

[24] L. Yin, X. Jin, W. Qiao et al., "PET imaging of brain function while puncturing the acupoint ST36," Chinese Medical Journal, vol. 116, no. 12, pp. 1836-1839, 2003.

[25] L. Schlünzen, M. S. Vafaee, and G. E. Cold, "Acupuncture of LI-4 in anesthetized healthy humans decreases cerebral blood flow in the putamen measured with positron emission tomography," Anesthesia and Analgesia, vol. 104, no. 2, pp. 308-311, 2007.

[26] G.-r. Shapo, L. Song, and W. Zhang, "PET/CT imaging of brain function during acupuncturing the acupoint ST36(zusanli)," Journal of Practical Radiology, vol. 07, pp. 890-892, 2007.

[27] L. Fang, T.-T. Ma, F. Zeng et al., "18F-FDG PET/CT study on the treatment of functional dyspepsia with acupuncture at meridian points," Shanghai Journal of Traditional Chinese Medicine, vol. 32, no. 3, pp. 157-161, 2013.

[28] Y. Huang, X.-S. Lai, J. Chen et al., "Glucose metabolism in different brain areas of vascular dementia patients after acupuncture at Baihui," Chinese Journal of Clinical Rehabilitation, vol. 36, pp. 68-70, 2005.

[29] Y.-W. Luo, A.-R. Tang, and D.-J. Li, "Application of PET imaging in acupuncture treatment of vascular dementia," Guangdong Medical Journal, vol. 12, pp. 2015-2016, 2008.

[30] X. Shi and F. Zuo, "Effects of acupuncture on glucose metabolism of stroke patients," Chinese Journal of Clinical Rehabilitation, vol. 10, no. 3, pp. 130-132, 2006.

[31] X.-Y. Zhang, S. Gao, J.-G. Zhao et al., "PET study of effects of combination of different points on glucose metabolism in the patient of cerebral infarction," Zhongguo Zhen Jiu, vol. 27, no. 1, pp. 26-30, 2007.

[32] P.-F. Shen and X.-M. Shi, "The clinical research on "The resuscitating" acupuncture method cure stroke patients 
observe the glucose metabolism," Chinese Archives of Traditional Chinese Medicine, vol. 28, no. 2, pp. 258-260, 2010.

[33] E.-T. Liu, S.-X. Wang, Y. Huang et al., "Effect of needling at waiguan (SJ5) on brain glucose metabolism in patients with cerebral infarction," Zhongguo Zhong Xi Yi Jie He Za Zhi, vol. 33, no. 10, pp. 1345-1351, 2013.

[34] F. Zeng, L. Lan, M.-L. Liu et al., "Cerebral responses to acupuncture at acupoints of gallbladder meridian in patients with functional dyspepsia," World Chinese Journal of Digestology, vol. 20, no. 23, pp. 2146-2150, 2012.

[35] Y.-X., Chen, D. Dai, T. Yu et al., “"Effect of acupuncture for "Reinforcing Qi and regulating blood to strengthen the root and tonify the kidney" on cerebral glucose metabolism of vascular dementia patients," Journal of Traditional Chinese Medicine, vol. 52, no. 13, pp. 1124-1127, 2011.

[36] Z. Sun, X.-S. Lai, and W. Shen, "Characteristics of PET functional imaging of acupuncture in stroke suffers at the acupoint sj5," Lishizhen Medicine and Materia Medica Research, vol. 23, no. 12, pp. 3145-3147, 2012.

[37] X.-S. Lai, Y. Huang, C.-Z. Tang et al., "A study on cerebral lobes. Glucose metabolism in vascular dementia patients treated by DU20, DU26 and HT7," Chinese Journal of Basic Medicine in Traditional Chinese Medicine, vol. 12, no. 5, pp. 375-377, 2006.

[38] X. Shi, F. Zuo, and J.-H. Tian, "Effects of electroacupuncture of baihui (GV 20)-qubin (GB 7) on glucose metabolism of cerebral motor function areas in stroke patients," Acupuncture research, vol. 3, pp. 167-170, 2005.

[39] X.-Z. Li, X.-G. Liu, W.-Z. Song et al., "Effect of acupuncture at acupoints of the Shaoyang Meridian on cerebral glucose metabolism in the patient of chronic migraine," Zhongguo Zhen Jiu, vol. 28, no. 11, pp. 854-859, 2008.

[40] M. Yang, J. Yang, F. Zeng et al., "Electroacupuncture stimulation at sub-specific acupoint and non-acupoint induced distinct brain glucose metabolism change in migraineurs: a PET-CT study," Journal of Translational Medicine, vol. 12, no. 1, p. 351, 2014.

[41] J. Yang, F. Zeng, Y. Feng et al., "A PET-CT study on the specificity of acupoints through acupuncture treatment in migraine patients," BMC Complementary and Alternative Medicine, vol. 12, no. 1, p. 123, 2012.

[42] F. Zeng, L. Lan, Y. Tang et al., "Cerebral responses to puncturing at different acupoints for treating meal-related functional dyspepsia," Neurogastroenterology \& Motility, vol. 27, no. 4, pp. 559-568, 2015.

[43] H. Zhang, X. Sun, S. Liu et al., "Neuronal activation by acupuncture at Yongquan (KI1) and sham acupoints in patients with disorder of consciousness: a positron emission tomography study," Neural Regeneration Research, vol. 9, no. 5, pp. 500-501, 2014.

[44] F. Zeng, W. Qin, T. Ma et al., "Influence of acupuncture treatment on cerebral activity in functional dyspepsia patients and its relationship with efficacy," American Journal of Gastroenterology, vol. 107, no. 8, pp. 1236-1247, 2012.

[45] Y. Huang, X. Jiang, Y. Zhuo, A. Tang, and G. Wik, "Complementary acupuncture treatment increases cerebral metabolism in patients with Parkinson's disease," International Journal of Neuroscience, vol. 119, no. 8, pp. 1190-1197, 2009.

[46] J. Pariente, P. White, R. S. J. Frackowiak, and G. Lewith, "Expectancy and belief modulate the neuronal substrates of pain treated by acupuncture," NeuroImage, vol. 25, no. 4, pp. 1161-1167, 2005.

[47] F. Zeng, W.-Z. Song, X.-G. Liu et al., "Brain areas involved in acupuncture treatment on functional dyspepsia patients: a PETCT study," Neuroscience Letters, vol. 456, no. 1, pp. 6-10, 2009.
[48] H. Zhang, W.-W. Chen, W.-Z. Song et al., "Brain glucose metabolism involved in acupuncture treatment on posttraumatic stress disorder patients," China Journal of Traditional Chinese Medicine and Pharmacy, vol. 25, no. 11, pp. 1882-1884, 2010.

[49] Y. Huang, A.-R. Tang, Q.-S. Li et al., "Effect of head acupuncture on glucose metabolism in temporal lobe of normal people and depressed patients," Jiangsu Journal of Traditional Chinese Medicine, vol. 25, no. 10, pp. 49-51, 2004.

[50] Y. Huang, A.-R. Tang, D.-J. Li et al., "Effect of head acupuncture on thalamic glucose metabolism in normal and depressed patients," New Traditional Chinese Medicine, vol. 36, no. 10, pp. 73-74, 2004.

[51] Y. Huang, A.-R. Tang, D.-J. Li et al., "Effect of head acupuncture on glucose metabolism in parietal lobe of patients with depression," Shandong Journal of Traditional Chinese Medicine, vol. 23, no. 9, pp. 543-545, 2004.

[52] Y. Huang, A.-R. Tang, Q.-S. Li et al., "Effect of head acupuncture on occipital glucose metabolism in normal and depressed patients," Yunnan Journal of Traditional Chinese Medicine, vol. 25, no. 4, pp. 37-72, 2004.

[53] L. Zhang, J.-L. Li, R.-Z. Yin et al., "Progress of study on the preparation of fluorine-18 labelled radiopharmaceuticals for brain receptor imaging using PET," Nuclear power in China, vol. 24, no. 5, pp. 428-432, 2001.

[54] M.-L. Liu, L. Lan, F. Zeng et al., "Quality control of the research on mechanisms of acupuncture therapy by using PETCT imaging techniques," Acupuncture Research, vol. 35, no. 1, pp. 67-70, 2010.

[55] X.-J. Xie, R.-X. Chen, Y. Fu et al., "Efficacy comparison of lumber disc herniation treated with mild moxibustion at Yaoyangguan (GV 3) under different conditions," Zhongguo Zhen Jiu, vol. 34, no. 11, pp. 1077-1080, 2014.

[56] X.-C. Yu, B. Zhu, J.-H. Gao et al., "The scientific basis of acupoint 26 dynamic process," Journal of Traditional Chinese Medicine, vol. 48, no. 11, pp. 971-973, 2007.

[57] C. J. Hogeboom, K. J. Sherman, and D. C. Cherkin, "Variation in diagnosis and treatment of chronic low back pain by traditional Chinese medicine acupuncturists," Complementary Therapies in Medicine, vol. 9, no. 3, pp. 154-166, 2001.

[58] F. Zeng, Z.-J. Li, Q. Liu et al., "Recommendations on acupuncture functional brain imaging," Liaoning Journal of Traditional Chinese Medicine, vol. 40, no. 2, pp. 373-375, 2013.

[59] T.-T. Ma, F. Zeng, Y. Li et al., "Which subtype of functional dyspepsia patients responses better to acupuncture? A retrospective analysis of a randomized controlled trial," Complementary Medicine Research, vol. 22, no. 2, pp. 94-100, 2015.

[60] C.-S. XU, C.-F. Li, J. Yang et al., "Effects of sample size on cerebral response to acupuncture with fMRI," Chinese Imaging Journal of Integrated Traditional and Western Medicine, vol. 9, no. 4, pp. 289-308, 2011.

[61] J. Suckling, A. Barnes, D. Job et al., "Power calculations for multicenter imaging studies controlled by the false discovery rate," Human Brain Mapping, vol. 31, no. 8, pp. 1183-1195, 2010.

[62] S.-B. Xu, B. Huang, C.-Y. Zhang et al., "Effectiveness of strengthened stimulation during acupuncture for the treatment of Bell palsy: a randomized controlled trial," Canadian Medical Association Journal, vol. 185, no. 6, pp. 473-478, 2013.

[63] J.-R. Chen, G.-L. Li, G.-F. Zhang, Y. Huang, S.-X. Wang, and N. Lu, "Brain Areas Involved in Acupuncture Needling Sensation of de qi: a single-photon emission computed tomography (SPECT) study," Acupuncture in Medicine, vol. 30, no. 4, pp. 316-323, 2012. 


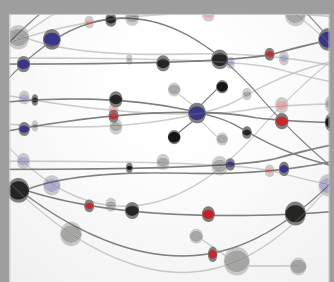

The Scientific World Journal
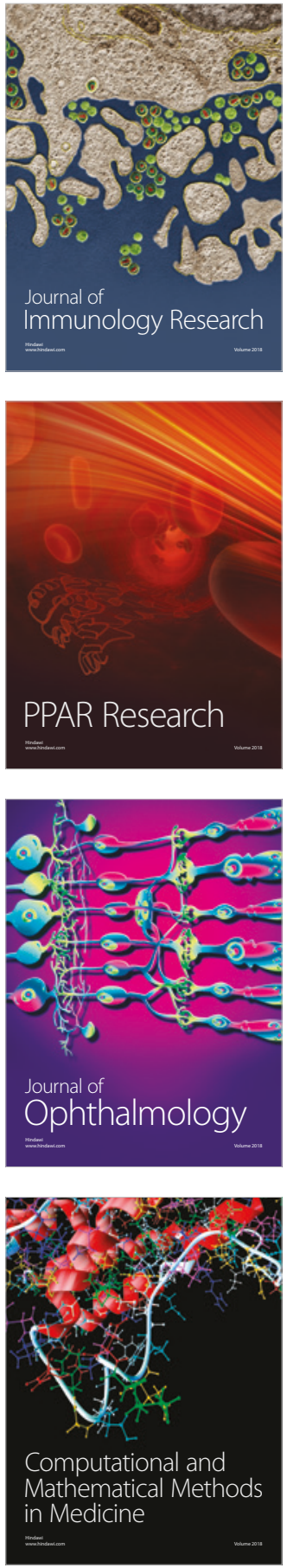

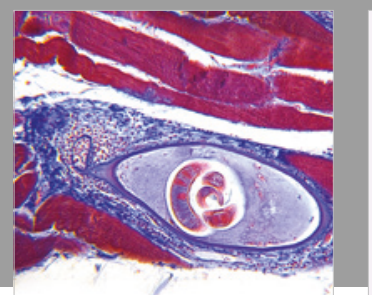

Gastroenterology Research and Practice

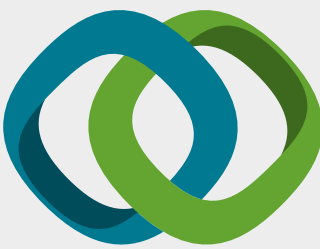

\section{Hindawi}

Submit your manuscripts at

www.hindawi.com
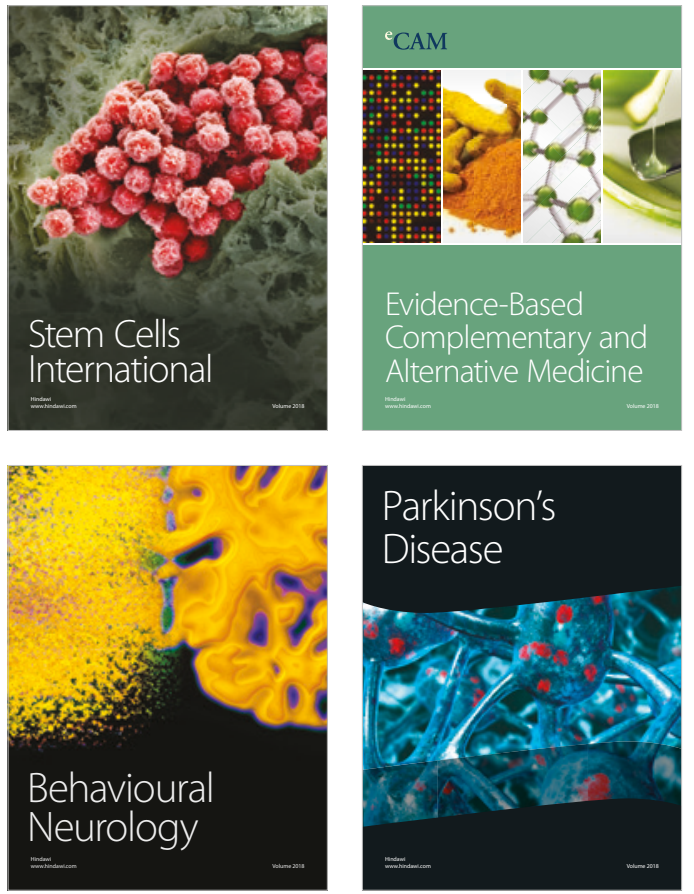

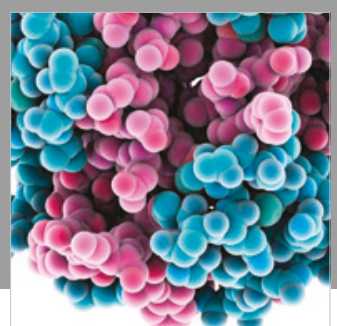

ournal of

Diabetes Research

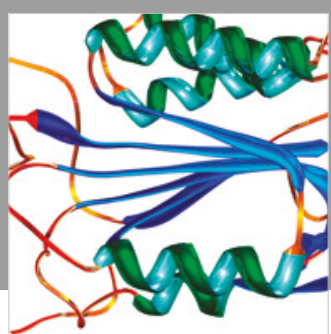

Disease Markers
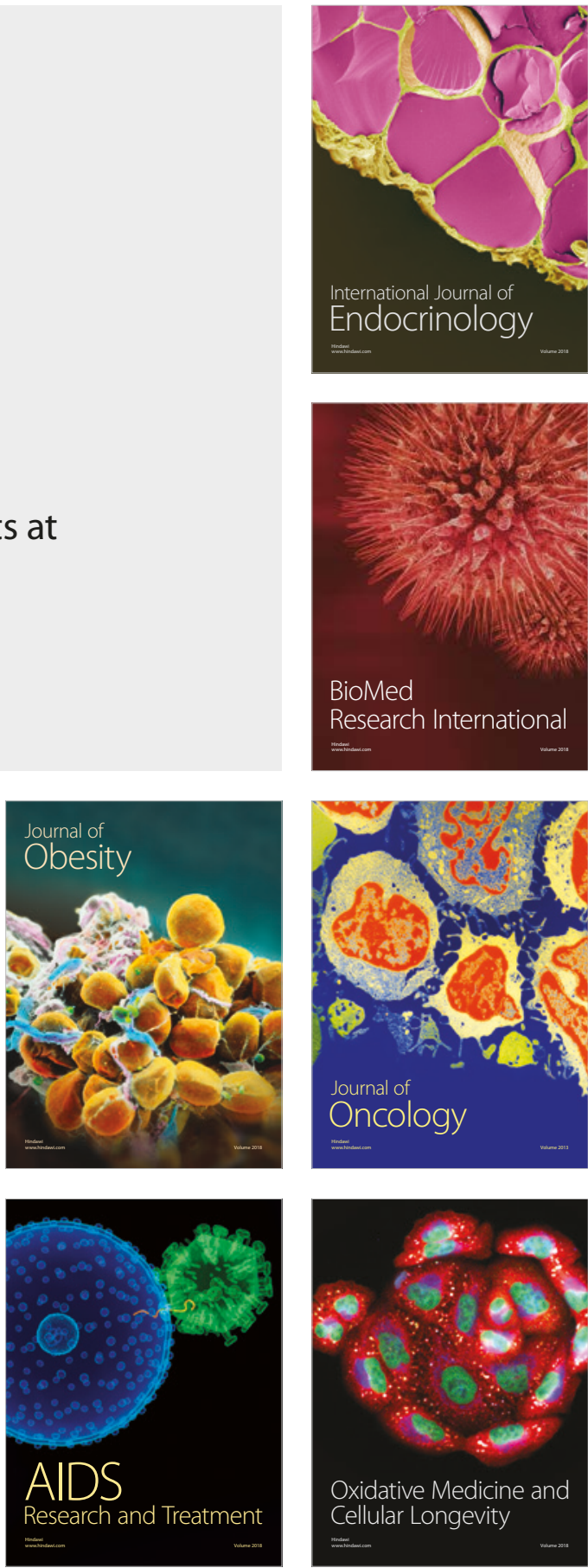